\title{
Learning Negotiation Skills: Four Models of Knowledge Creation and Transfer
}

\author{
Janice Nadler • Leigh Thompson • Leaf Van Boven \\ Northwestern University, School of Law, 357 East Chicago Avenue, Chicago, Illinois 60611, \\ and American Bar Foundation, Chicago, Illinois 60611 \\ Northwestern University, Kellogg School of Management, 2001 Sheridan Road, \\ Evanston, Illinois 60208-2001 \\ University of Colorado at Boulder, Department of Psychology, Muenzinger Hall, Box 345, \\ Boulder, Colorado 80309 \\ jnadler@northwestern.edu • leighthompson@kellogg.northwestern.edu • vanboven@psych.colorado.edu
}

\begin{abstract}
$\mathrm{O}$ ur review of the learning and training literature revealed four common methods for training people to be more effective negotiators: didactic learning, learning via information revelation, analogical learning, and observational learning. We tested each of these methods experimentally in an experiential context and found that observational learning and analogical learning led to negotiated outcomes that were more favorable for both parties, compared to a baseline condition of learning through experience alone. Information revelation and didactic learning were not significantly different from any other condition. Process measures revealed that negotiators' schemas about the task (reflected in open-ended essays) were strong predictors of performance in the analogical learning condition, but were poor predictors of performance in the remaining conditions. Interestingly, negotiators in the observation group showed the largest increase in performance, but the least ability to articulate the learning principles that helped them improve, suggesting that they had acquired tacit knowledge that they were unable to articulate.
\end{abstract}

(Negotiation; Learning; Analogical Reasoning; Management Skills)

\section{Introduction}

Organizational learning occurs at several different levels. At the individual level, people in organizations are called upon to transfer knowledge and skills learned in one setting to novel situations. At the interpersonal level, this type of individual learning or transfer often occurs in the context of social relationships, teams, groups, or dyads. Moreover, organizations often influence or are influenced by individual and interpersonal learning (Argyris 1993). This paper focuses on individual learning in the context of mixed-motive dyadic relationships.

\section{Types of Learning}

In the present research, we examined the efficacy of four types of learning methods on the ability of people to improve their negotiation skills. Building upon prior research on the role of different kinds of experience in negotiation performance (e.g., Thompson 1990a, 1990b), we tested a baseline condition of experience alone against four different learning methods that were combined with experience. A recent survey of negotiation training across four disciplines-management, law, education, and policy-revealed that negotiation instructors rely on experiential learning and simulations as a teach- 
ing tool (Harvard Law School Program on Negotiation) (www.pon.harvard.edu〉. The specific nature of experiential learning of negotiation skills, however, varies dramatically across schools, programs, and classes.

Our review of the research literature in negotiation reveals at least four ways in which experiential learning is implemented: didactic learning (or principle-based learning), learning via information revelation, analogical learning, and observational learning. By combining each of these four methods with simulations and exercises, many variations on experiential learning are both conceptually plausible and relevant in practice. To our knowledge, however, researchers have not compared the effectiveness of these learning techniques by experimentally testing them.

The experiment reported here extends research and theory by demonstrating substantial performance differences in negotiation that are attributable to the methods by which negotiators learned to negotiate. The goal of this paper, therefore, is to test the relative efficacy of different learning methods in negotiation, rather than to gain a thorough understanding of the various and complex cognitive mechanisms underlying each method. We briefly discuss the theoretical underpinnings and empirical evidence for each of these learning techniques. We review the key underlying mechanisms that guide each type of learning, with a particular focus on examining learning in negotiations.

Principle-Based Learning. Also known as didactic learning, this type of learning is widely used as an educational tool in negotiation. For example, students are often told how they can take advantage of varying preferences to "expand the pie." However, novices are often unable to appreciate and extend principles to novel situations (Novick 1988). Further, unless there is a close connection between a principle and relevant examples, people are unable to take advantage of abstract principles (Ross and Kilbane 1997). According to Neale and Northcraft (1990), negotiators need to have a strategic conceptualization of the bargaining situation to reach integrative solutions. In an investigation by Bazerman and Neale (1982), some negotiators were given training designed to decrease negotiator overconfidence-a major impediment to effective negotiations. The training decreased overconfidence. However, other investigations reveal that providing learners with a principle is not as effective as other types of learning, such as analogical learning (Gick and Holyoak 1983, Ross and Kilbane 1997, Loewenstein et al. 1999).

Learning via Information Revelation. Behavioral decision theory suggests that experience with a task should improve judgment and performance (Einhorn and Hogarth 1978, Hogarth 1981). According to this perspective, suboptimal negotiation performance primarily occurs in isolated or discrete decision situations characterized by an absence of information about the structure of the task or problem. When the environment returns the type of information necessary to compare the present strategy with a representation of an ideal strategy, then performance can improve (Balzer et al. 1989, Hammond et al. 1980). Einhorn and Hogarth (1978) developed a judgmentaction-outcome model to represent the self-correcting function of feedback. According to the model, outcomes follow from people's initial judgments and predictions, which are then used to determine actions and choices. Outcomes produce two feedback mechanisms: a direct effect on subsequent judgments and actions, and an indirect effect based upon a person's interpretation or evaluation of the outcomes. Access to the other party's information about their priorities and preferences in a prior negotiation allows negotiators to correct their interpretation of the negotiated agreement. Thompson and DeHarpport (1994) found that revealing information about the opponents' priorities and preferences at the conclusion of a completed negotiation resulted in superior transfer, or performance on a subsequent negotiation task, compared to providing outcome information alone. Similarly, Ball et al. (1991) provided only outcome information revelation (money gained or lost as a result of bidding decisions) and found that this was not effective. And Balke et al.'s (1973) investigation of social judgment theory in reenacted labor-management negotiations revealed that when negotiators were given cognitive information revelation about the opponent's interests, they were more likely to reach consensus. For the purposes of the 
present investigation, we selected the most causally efficacious aspects of these experiments to create a learning treatment that provided information about the opponent's priorities and preferences, as well as the outcome of the completed negotiation.

Analogical Learning. Research on analogical processing shows the importance of analogy in learning and understanding (Holyoak and Thagard 1995). Students can often understand a novel situation by transferring knowledge from a well-understood situation (Polya 1957, Pirolli and Anderson 1985). Ross (1987) found that giving learners analogical examples to illustrate a probability principle facilitated their later use of the probability formula to solve other problems. The standard approach to analogical learning involves mapping information from a base-a well-understood domain that serves as the information source-to a less familiar target (Gentner 1989). The target problem is typically a different, novel problem that is from a different domain, thus appearing on the surface to have little or nothing to do with the first problem. However, the underlying structure or "deep structure" of the problems may be quite similar. The critical question is: What are the conditions that allow the learner to "recognize" the applicability of the old problem in this new domain? According to Gentner's (1983) structure-mapping theory, analogical reasoning involves setting up correspondences between structured representational elements of two domains and transferring information guided by the common relational structure (Gentner 1983, 1989, Holyoak 1985, Holyoak and Thagard 1989, 1997). In this sense, structure-mapping theory involves a comparison between source cases or between source and target cases, such that the underlying relational structure becomes obvious. Thus, making comparisons can inform students and managers as to which aspects of experience are relevant and which are causally irrelevant.

In a previous test of structure-mapping theory, we used a simple manipulation, designed to induce analogical reasoning (Thompson et al. 2000, Loewenstein et al. 1999). Prior to a real face-to-face negotiation, we gave students two mininegotiation cases (approximately 200 words). Each case described how a protagonist effectively solved a difficult and vexing negotiation situation by using a particular negotiation strategy, such as a contingent contract. (A contingent contract is a negotiation strategy in which parties resolve differences via betting on the future (see Bazerman and Gillespie 1999). Some students were told to analyze each case and identify the key principle in each. Other students were told to compare the two cases, with the theory being that comparison allows students to abstract a common denominator that is not tied to irrelevant surface aspects of the situation (Gentner et al. 1993). Later (sometimes minutes, sometimes a week), students were challenged with a real, face-to-face, one-on-one negotiation situation in which a contingent contract could be used to add considerable individual value. On average across our studies, 50 to $60 \%$ of students in the "compare" group used contingent contracts in the face-to-face situation; less than half that amount used contingent contracts in the "separate cases" group (Thompson et al. 2000, Loewenstein et al. 1999). Process data revealed that analogical comparison of the cases encouraged recognition of a problem's structural features, suggesting that analogy facilitates transfer.

Further, analogy facilitates creativity and flexibility when negotiating. Spector (1997) briefed students on an international treaty dispute, and then instructed them to think about the case using either analogy (e.g., "write down as many analogies as you can think of") or standard case analysis (e.g., "inventory the issues, positions, and interests of the parties"). Afterwards, students were randomly assigned to represent one of the countries in the treaty dispute as a scientific expert, and were instructed to negotiate with a counterpart to find a solution to the conflict. Participants trained in analogy were less likely to reach impasse than negotiations in which participants had standard training. Further, participants trained in analogy were more likely than their standard training counterparts to change their perceptions of the best perceived solution during the course of training and negotiation, demonstrating an ability to adjust goals in response to new information. In sum, whereas previous research demonstrates that analogy more effectively facilitates transfer than case analysis, these studies have not 
compared the effectiveness of analogy training with the other methods of learning that we discuss here.

Observational Learning. Also known as imitation or modeling, observational learning is based on the premise that negotiators can improve their own skills by observing those of others. Specifically, by watching a "model" negotiator conduct a negotiation in an integrative fashion, negotiators might be better prepared to conduct their own negotiations. Many complex social behaviors, such as aggression, helping, and gender-appropriate behavior are successfully learned by observing others (Bandura 1973, Bryan and Test 1967). According to social learning theory, social feedback provides people with information about which behaviors are likely to be rewarded. Bandura's (1973) portrayal of the psychological mechanisms involved in observational learning focuses on symbolic acquisition, such that observers do not simply mimic a model's actions through reinforcement-based principles of stimulus-response association. Instead, observers are active processors of the information provided by a model's behavior. Bandura's (1973) model contains four key components of observational learning: attentional processes, retention processes, motor reproduction processes, and motivational processes. Observational learning is facilitated when the observer pays attention to the model, retains the information, has the capacity to enact the model's behaviors, and is motivated to behave. In developing our observational learning treatment, we attempted to maximize each of these critical processes.

Evidence from research on learning in contexts other than negotiation suggests that observation produces efficient, effective learning. Shebilske et al. (1998) demonstrated that students performing a pilot training video task improved their performance from observing another student doing the same task. In addition, students show greater improvement in writing skills after having observed another person thinking aloud while writing, as compared to simply practicing writing on their own (Couzijn 1999). Finally, brain scans of people observing hand actions of another person (the target) are revealing as well. When the target performed meaningful actions, the left hemisphere was engaged; when the target performed meaningless actions, the right occipitoparietal pathway was engaged. Further, when participants were told they would have to recognize the hand actions later, memory and encoding structures were activated; when participants were told they would have to imitate the hand actions later, planning and action generation regions were activated (Decety et al. 1997). This suggests that when negotiators observe a model's actions with the goal of subsequent imitation, they process the information they observe in a way that will be meaningful during the imitation stage. We hypothesize that in a bargaining context, negotiators who observe a model negotiation are likely to notice and imitate the behaviors that lead to efficient distribution of resources.

\section{Overview of Present Research}

We report an experiment that was designed to examine and compare the effectiveness of the four types of learning just discussed. We followed the tradition of Cook and Campbell (1979) and Platt (1964), such that we developed "strong" treatments with the goal of determining which of the learning treatments was most effective. We created four different learning conditions, corresponding to the types of learning just reviewed: didactic, information revelation, analogical, and observational. We report a study in which all negotiators first engaged in an initial negotiation with no formal learning. Then, we randomly selected a baseline group of negotiators who would receive no further training and compared their performance with negotiators who would add one of four types of formal training to their negotiation experience, much as would be the case in a classroom or corporate training session.

We also compared each of the learning treatments to a pure experiential learning baseline condition. Our key measure of learning was performance on the task. We designed each of the learning treatments to instruct about one particularly important aspect of negotiation-integrative bargaining. We were careful to use performance on the first task as a covariate so that any differences would be attributable to the experimental treatments, not arbitrary differences on the first task. To test the extent to which each learn- 
ing method led to successful articulation of the principles learned therein, we also included an open-ended process check measure in which negotiators wrote in an open-ended fashion about their reflections of their own learning (cf. Thompson and DeHarpport 1994).

\section{Method}

\section{Participants}

A total of 122 undergraduate psychology students volunteered to participate and were each paid \$15 for their participation. Precautions were taken to ensure that participants within a dyad did not previously know each other.

\section{Procedure}

Participants were randomly assigned to experimental conditions and bargaining roles, and were informed that they would be randomly paired with another person with whom they would engage in two negotiations. In each negotiation, dyads bargained for a maximum of 25 minutes. As an incentive, participants were informed that, for each of the two negotiation tasks, one $\$ 100$ cash prize and ten $\$ 20$ cash prizes would be awarded at the end of the semester. The probability of winning a prize corresponded to their performance in the negotiation (see Thompson and Hastie 1990).

\section{Negotiation Tasks}

For both negotiation tasks, participants were given instructions specific to their role, a payoff schedule, and a short quiz to ensure that they understood the task. The payoff schedules for both tasks are presented in the Appendix. Negotiators bargained face to face with no restrictions on communication, except that they were told not to physically exchange their payoff schedules.

Two different tasks were used; each task was unique in that different patterns of trade-offs and alternatives were necessary to reach a fully integrative agreement. The two tasks differed in terms of the content and setting of the negotiation and the issues to be negotiated. The first task was a job contract negotiation between an employee and employer, involving issues of salary, starting bonus, job assignment, insurance coverage, and moving expenses. The second task was more complex and involved a commercial real estate negotiation between a development company and a city planner's office.

Each task contained different types of issues to be negotiated. Thus, generalizing from the first to the second task was not easy; it required that participants have an understanding of the nature of the situation. For example, the second task included one purely fixed-sum (distributive) issue in which gains for one person result in equal losses for the other party; the first task did not include a distributive issue. In the second task, there was one pair of variable sum (logrolling) issues in which gains for one person do not represent equal losses for the other; in contrast, the first task had two pairs of logrolling issues. Each task involved one compatible issue in which both negotiators had identical preferences. ${ }^{1}$ Participants were told that they should try to accumulate as many points as they could in each negotiation, and that failure to reach an agreement would result in both persons earning zero points.

\section{Experimental Conditions}

The independent variable was between participants, and involved implementation of one of four different learning methods after the completion of the first negotiation (but before beginning the second negotiation). The four learning conditions were: didactic, information revelation, analogical, and observational. Participants in the baseline or control condition completed the two negotiation tasks without any instruction in between. Dyads were randomly assigned to one of the five conditions.

Baseline Condition. In the baseline condition, participants completed the first negotiation task and were then asked to complete the process check measure (described later). Then, they were introduced to the second negotiation task, following which they completed the judgment measure for that task.

\footnotetext{
${ }^{1}$ We performed analyses on compatible issues, but did not note any effects of the experimental treatments. In the interest of brevity, these measures are not further discussed.
} 
Didactic. In the didactic condition, participants were given a one-page synopsis of key principles of negotiation taken from a textbook. The key principles were identified and labeled: trade-offs among differentially weighted issues and the existence of compatible issues. The synopsis was edited to remove all illustrations and examples, so that the didactic condition would remain conceptually distinct from the analogy condition. Participants were asked to study the synopsis and then to complete the process check measure.

Information Revelation. In the information revelation condition, participants were given complete information about the other person's payoff schedule during the negotiation. The participant's own payoff schedule was printed on the same page so that participants could clearly see the underlying structure of the negotiation that they had just completed (although it was not explicitly pointed out to them). Participants were asked to study the payoff schedules and then complete the process check measure.

Analogical. In the analogical condition, participants were given two short vignettes to read (approximately 200 words each), each describing a negotiation situation. Whereas the surface features in the stories were quite different, the underlying deep structure of the vignettes were similar. The two vignettes described situations in which parties recognized opportunities for trade-offs (however, the word "trade-off" was not explicitly used). After reading the stories, participants were asked to complete the process check measure.

Observational. In the observational condition, participants were shown a videotaped version of the negotiation they had just completed in which the negotiators (portrayed by actors) reached a fully integrative (win-win) agreement. The videotape portrayed the negotiators exchanging information about the issues that were most and least important to each of them. Negotiators discussed more than one issue at a time, and traded concessions on issues considered least important in exchange for achieving preferences on issues considered most important to each respec- tive negotiator. After watching the videotaped negotiation, participants were asked to calculate the score of the target negotiator who played their role and then to complete the process check measure.

\section{Dependent Measures}

Negotiation Performance. For each negotiation, a joint performance score was assigned to each dyad, summing both negotiators' total scores in the negotiation. In addition, a trade-off score was computed to measure the extent to which negotiators maximized joint gain by trading off issues. In the first negotiation, trade-off scores were computed by summing the points negotiators earned on the four issues for which negotiators had different preferences (signing bonus, moving expense coverage, insurance coverage, and salary; see Appendix). In the second negotiation, trade-off scores were computed by summing the points earned on the two issues for which negotiators had different preferences (local subcontractors and building height; see Appendix).

Process Check Measure. As a check on understanding of the learning process just experienced, each participant was asked to express his or her observations following the learning manipulation in each condition. To this end, each person in each condition was asked to write down his or her observations about the task in an open-ended fashion. We used an established coding scheme (Thompson and DeHarpport 1994, Thompson et al. 2000) to rate the quality of participants' understanding in each condition. Two trained raters evaluated the quality of each participant's written observations using a 3-point scale: $1=$ no understanding of the nature of the task (e.g., "Basically, we stated what we wanted and then compromised to a value in the middle"), $2=$ some understanding of the nature of the task (e.g., "The job assignment was agreed upon quickly because we both wanted the same thing"), and $3=$ a full understanding of the nature of the task (e.g., "I asked her what she felt was most important .... She seemed agreeable to having only $\$ 32 \mathrm{~K}$ for her salary if she had a $90 \%$ moving coverage, and point-wise this was good for me, 
so I quickly agreed"). Interrater reliability (assessed using Cohen's Kappa) was 0.81. Scores within dyads were summed to form a joint score. Thus, the possible range dyad scores on the analysis of the subjective learning measure was 2 through $6 .^{2}$

\section{Results}

\section{Negotiation Performance}

Joint Outcome. An analysis of covariance (ANCOVA) was performed to examine the impact of the learning treatments on the key measure of learning: joint outcomes in the second negotiation. Joint scores in the second negotiation-which involved fewer issues and a different point scale than the first negotiation-ranged from 820 to $1,120^{3}(M=1,014$, $s d=98) .{ }^{4}$ Because the learning manipulation occurred after the first negotiation but prior to the second negotiation, we used the joint outcome for each dyad in the first negotiation as a covariate (which did not significantly differ between conditions, $F<1$ ). The ANCOVA revealed that the learning manipulation had a significant overall effect on joint outcome in the second negotiation, $F(4,57)=2.61, p<0.05$.

The bars in Figure 1 represent the adjusted mean joint outcome for each experimental group in the second negotiation. Post hoc comparisons revealed that the adjusted mean joint scores in the second negotiation were significantly higher in the observational (Adj. $M=1,068.07, s d=41.01$ ) and analogical (Adj. $M=1,057.82, s d=62.93$ ) treatment conditions, as compared with the baseline experiential condition (Adj. $M=969.49$, $s d=98.12$ ), $F(1,20)=11.43 ; p<0.01$; $F(1,19)=9.48 ; p<0.01$, respectively. The informa-

\footnotetext{
${ }^{2}$ In the interests of brevity, this article omits certain measures collected in the experiment. These results are available from the authors upon request.

${ }^{3}$ We express joint scores in the second negotiation in 1,000s; e.g., a joint score of 820 in the second negotiation amounts to a score of $\$ 820,000$.

${ }^{4}$ Three dyads were omitted from all analyses: one dyad failed to reach an agreement; two dyads reached agreements whose values were extreme, differing from the mean by two standard deviations.
}

Figure 1 Effect of Learning Condition on Final Joint Outcome and Trade-off Score, Second Negotiation

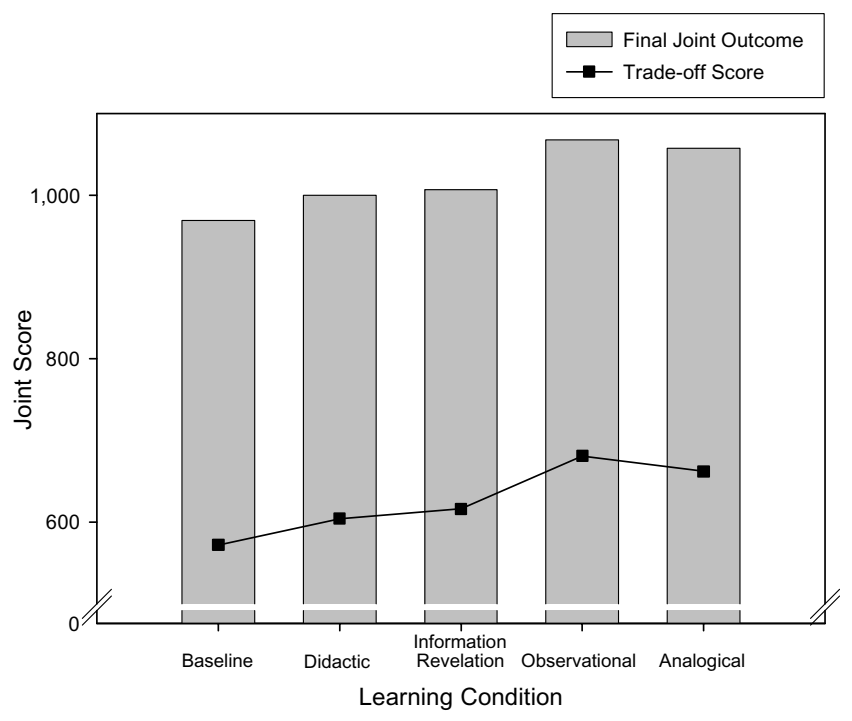

tion revelation (Adj. $M=1,006.94, s d=107.22$ ) and didactic (Adj. $M=1,000.50, s d=118.97$ ) groups were not significantly different from the baseline and analogical groups (all $F^{\prime} \mathrm{s}<3.0, p^{\prime} \mathrm{s}>0.10$ ). There were marginally significant differences between the observational and didactic conditions $(F(1,20)=3.53 ; p=$ $0.075)$ and between the observational and information revelation conditions $(F(1,20)=3.41 ; p=0.08)$. Thus, while not every pairwise comparison revealed statistically significant mean differences, there was improvement in negotiation performance when experiential learning (baseline condition) was supplemented with observational or analogical learning.

Identifying Trade-offs. The trade-off score reflected the degree to which parties traded off the two issues for which they had different prioritiese.g., negotiators who "split the difference" on the number of local subcontractors and building height (two subcontractors and four stories) had lower tradeoff scores than did negotiators who traded off these two issues (four subcontractors and six stories; see Appendix). In the first negotiation, only $3 \%$ of all dyads achieved a perfect trade-off score of 2,000. Trade-off scores on the second task ranged from 420 
to 720 (the possible range was 240 to 720$)(M=$ $624.7, s d=95.2)$. In the second negotiation, $37 \%$ of all dyads achieved a perfect score of 720 , representing a significant improvement over first negotiation trade-off scores, $\chi^{2}(1)=20.03, p<0.001$. An examination of dyads in the baseline condition revealed that while $0 \%$ of the dyads achieved a perfect tradeoff score in the first negotiation, $25 \%$ achieved a perfect score in the second negotiation, $\chi^{2}(1)=3.43, p=$ 0.064 . This suggests that negotiators' performances improved with experience, although the difference is only marginally significant.

In the second negotiation, did negotiators who received training outperform those who received no training? To find out, we conducted an ANCOVA to examine the impact of learning manipulation on trade-off scores in each condition. The trade-off score for each dyad in the first prelearning negotiation was used as a covariate; as expected, there were no between-group differences in trade-off scores for the first negotiation (which occurred prior to the treatment), $F<1$.

The pattern of trade-off scores in the second negotiation mirrored that found for joint outcomes. The learning variable had a significant overall effect on trade-off scores in the different groups, $F(4,57)=2.61$, $p<0.05$. The line graph in Figure 1 illustrates the adjusted mean trade-off score for each experimental group in the second negotiation. Post hoc comparisons revealed that the adjusted mean trade-off scores in the second negotiation were significantly higher in the observational (Adj. $M=681.02, s d=46.65$ ) and analogical (Adj. $M=662.02, s d=61.64$ ) treatment conditions, as compared with the baseline experiential condition (Adj. $M=571.74, s d=98.12) ; F(1,20)=$ 12.54; $p<0.01 ; F(1,19)=8.93 ; p<0.01$, respectively. The information revelation (Adj. $M=615.93$, $s d=$ 105.74) and didactic (Adj. $M=603.88, s d=111.12$ ) groups were not significantly different from other groups. These results suggest that the effectiveness of the observational and analogical conditions for overall performance is primarily due to the trade-off strategy.

\section{Process Check}

Following the learning manipulation, participants wrote an open-ended essay about their observations of the task. These responses were coded by two trained assistants. Some participants failed to write anything when prompted to write a summary paragraph, resulting in the omission of one dyad from the didactic condition and one dyad from the analogical condition. Interrater reliability (assessed using Cohen's Kappa) was 0.81 .

The type of learning treatment affected the quality of the opened-ended essays, $F(4,53)=7.03$, $p<0.0001$. Post hoc comparisons (Tukey's Honestly Significantly Different (HSD); $p<0.05)$ showed that the analogical, information revelation, and didactic learning groups had significantly higher scores $(M=4.41, s d=0.67 ; M=4.10, s d=0.93 ; M=4.63$, $s d=0.91$, respectively) than did the baseline learning $(M=3.67, s d=0.89)$, and observational learning $(M=3.05, s d=0.43)$ groups. More specifically, participants in the analogical and didactic learning groups were able to articulate the key underlying structure of the task well, whereas participants in the baseline and observational groups were not. Put another way, whereas only a small percentage of dyads in the baseline (33\%), information revelation (33\%), and observational $(0 \%)$ groups were able to articulate their respective learning principle to a fair degree (a combined score of more than 4.0), an overwhelming majority of dyads in the didactic $(67 \%)$ and analogical $(73 \%)$ were able to do so. Negotiators in the analogical treatment group also performed quite well in terms of joint outcome in the second negotiation, suggesting that they were able to transfer the new concepts demonstrated in the process check exercise into practice in the subsequent negotiation. Negotiators in the didactic learning group, however, did not perform well in the second negotiation, suggesting that their ability to articulate principles did not transfer well to putting those principles into practice.

\section{Discussion}

The purpose of this experiment was to examine the relative efficacy of different types of training techniques for improving negotiation skills. Participants who received observational training or analogical training performed better in negotiations than did 
participants who only received baseline (experiential) training. Didactic training and training via information revelation were not significantly different from the other groups. The participants in the observational and analogical groups were more likely to construct profitable trade-offs among issues than were participants in the baseline group. However, contrary to prior research (Thompson et al. 2000, Loewenstein et al. 1999), we did not find significantly different negotiation performance between the analogical and didactic groups, although the pattern of means was consistent with prior research.

We complemented this measure of learning with a quite different measure that was unstructured. We simply asked participants to write down in their own words, in an open-ended fashion, their observations about the negotiation. It was clear that some participants' essays were quite remarkable in "capturing" the important, underlying essence of the negotiation (e.g., "I gave up in a category to get more in another category" - reflecting the important idea of a tradeoff). In contrast, other participants focused on irrelevant factors and strategies that, in general, were not effective (e.g., " ... we each stated what we wanted and then compromised to a value more or less right in the middle of the two").

A more detailed examination of the individual learning conditions revealed that negotiators in the information revelation condition excelled at accurately inferring the other party's interests. Negotiators in this condition received information about their opponent's priorities and preferences after the first negotiation. However, this was not sufficient for achieving substantial improvement in performance. Apparently, negotiators who received information about the other party's priorities and preferences understood the information, but were unable to transfer this knowledge and apply it to a new negotiation. Similarly, negotiators in the didactic group wrote complex and meaningful observations about the negotiation, yet did not distinguish themselves when actually seated at the bargaining table. It is reasonable to think that the didactic learning group might have simply parroted back the concepts they were exposed to, but did not transfer them to the next negotiation task. Negotiators in the analogical learning group excelled in the process check, writing complex and meaningful observations about the negotiation experience. Unlike negotiators in the information revelation and the didactic groups, however, negotiators in the analogical condition apparently were able to transfer this knowledge to a new negotiation setting in which their performance excelled.

One puzzling result was the performance of negotiators in the observational condition, who excelled at the second negotiation, and yet performed miserably on the process check essay ( $0 \%$ articulated relevant learning principles to a fair degree). Apparently, the process of observing other people negotiating improved the performance of the observational group negotiators in the subsequent negotiation, but they were almost entirely unable to articulate the learning principles that helped them to improve. One possible explanation for this perplexing result is that negotiators in the observational condition had acquired tacit knowledge (Polanyi 1958) that improved their performance. Thus, their improved performance in the second negotiation was attributable to new skills they had acquired but were unaware of having, and unable to articulate in the written essay.

\section{Conclusion}

In this discussion, we raise the following theoretical and practical issues: (1) Why is analogical and observational training particularly effective? and (2) What can and should be done regarding the future of research on learning in management contexts?

Contrary to popular intuition, simply having experience-in the absence of information revelation, principles, observation, or drawing analogies to other cases-is largely ineffective. The limitations of this type of learning are implicitly recognized in the vast amounts of money spent on the education of experienced, successful professionals wishing to improve their negotiation skills. Indeed, negotiators in the baseline experiential condition were not accurate in assessing the task, nor were their subjec- 
tive observations particularly complex or meaningful. The analogical group was remarkably effective at improving their performance, as a consequence of drawing analogies to seemingly unrelated negotiation situations. We were careful to provide narratives that were devoid of any mention of principles. Further, we were careful to use narratives that had virtually no similar surface features to the face-toface negotiations that participants actually completed. Thus, negotiators in the analogical group could not have been relying on simple surface similarities. They also wrote elegant open-ended essays, suggesting that the case stories they had read helped them to form more complex thoughts about the negotiation situation. The effects for the analogical learning group provide greater generalizability in terms of the integrative outcomes for the growing body of research on analogical reasoning in negotiation. Prior research has nearly exclusively focused on the ability of negotiators to develop contingency contracts (cf. Thompson et al. 2000, Loewenstein et al. 1999); the present research extends these effects to the important skill of trade-offs.

Participants in the information revelation condition are comparable to the descriptive-trained participants in Donnelly and McDaniel (1993), in that these participants received a quantitative description of the opponent's preferences and interests after the first negotiation. However, putting this knowledge into action at the table was not easy, even under apparently ideal conditions: one's negotiation partner had the same (accurate) understanding of the task, and there was virtually no time delay between the tasks.

If anything, it is the observational condition participants who remain the most elusive. They achieved the highest outcomes, yet their process measure scores were not impressive. They were apparently learning something useful that they could not readily articulate. Watching a reenactment of a negotiation conducted by skilled negotiators was remarkably effective, even though no formal concepts or strategies were mentioned in the tape. Moreover, because the second negotiation differed so much from the first negotiation, it would not have been possi- ble for participants to blindly imitate the modelsthe issues were different in name, number, and type.

We fully recognize that our experimental conditions reflected "pure" types, whereas most learning as it occurs in management classrooms is often a hybrid. However, hybrid models often develop in the absence of knowledge of the effectiveness of their components. Examination of "pure" types is an important precursor to examination of hybrid models. We also recognize, as mentioned earlier, that our manipulations are "strong," incorporating relatively heavy-handed implementations of different types of learning. Just as examination of "pure" types of learning is an important precursor to hybrid types of learning, an examination of strong implementations of learning are important precursors to examinations of weaker learning manipulations. An obvious next step is to examine how blends of the four major approaches studied in the present investigation might fare in terms of actual effectiveness. Indeed, it is our supposition that more often than not, hybrid models of teaching and training are more extensively used than the pure models.

In an era in which management has become increasingly focused on knowledge, learning, and continuous improvement, it is of paramount importance to understand the mechanisms by which managers learn and develop new skills. The "new look" at knowledge and learning in organizations focuses on knowledge implementation, as opposed to knowledge acquisition. The results of the present investigation suggest that the ability to put knowledge into meaningful action is not as straightforward as previously believed and that the manager may best serve his or her own professional development and the success of his or her organization via incorporating principles of cognitive-social psychology.

\section{Acknowledgments}

The authors thank Linda Argote, Bill McEvily, and Ray Reagans for helpful comments. This research was supported by the Dispute Resolution Research Center at Northwestern University, the American Bar Foundation, and the National Science Foundation Grant 9870892. 
NADLER, THOMPSON, AND VAN BOVEN

Knowledge Creation and Transfer in Negotiation

\section{Appendix}

Task 1

\begin{tabular}{|c|c|c|}
\hline Issue & Employee points & Employer points \\
\hline \multicolumn{3}{|c|}{ Signing bonus } \\
\hline $10 \%$ & 600 & 0 \\
\hline $8 \%$ & 450 & 60 \\
\hline $6 \%$ & 300 & 120 \\
\hline $4 \%$ & 150 & 180 \\
\hline $2 \%$ & 0 & 240 \\
\hline \multicolumn{3}{|c|}{ Job assignment } \\
\hline Division A & 0 & 0 \\
\hline Division B & 30 & 30 \\
\hline Division C & 60 & 60 \\
\hline Division D & 90 & 90 \\
\hline Division $\mathrm{E}$ & 120 & 120 \\
\hline \multicolumn{3}{|c|}{ Moving expense coverage } \\
\hline $100 \%$ & 400 & 0 \\
\hline $90 \%$ & 300 & 20 \\
\hline $80 \%$ & 200 & 40 \\
\hline $70 \%$ & 100 & 60 \\
\hline $60 \%$ & 0 & 80 \\
\hline \multicolumn{3}{|c|}{ Insurance coverage } \\
\hline $100 \%$ & 80 & 0 \\
\hline $80 \%$ & 60 & 100 \\
\hline $60 \%$ & 40 & 200 \\
\hline $0 \%$ & 20 & 300 \\
\hline $20 \%$ & 0 & 400 \\
\hline \multicolumn{3}{|l|}{ Salary } \\
\hline$\$ 40,000$ & 240 & 0 \\
\hline$\$ 38,000$ & 180 & 150 \\
\hline$\$ 36,000$ & 120 & 300 \\
\hline$\$ 34,000$ & 60 & 450 \\
\hline$\$ 32,000$ & 0 & 600 \\
\hline
\end{tabular}

Task 2

\begin{tabular}{lrr}
\hline Issue & Total value to Bainbridge & Total value to Alki \\
\hline $\begin{array}{l}\text { Number of local subcontractors } \\
4\end{array}$ & $\$ 360,000$ & \\
3 & $\$ 240,000$ & $\$ 0$ \\
2 & $\$ 180,000$ & $\$ 30,000$ \\
1 & $\$ 120,000$ & $\$ 60,000$ \\
0 & $\$ 0$ & $\$ 90,000$ \\
Percent of open space & & $\$ 120,000$ \\
$30 \%$ & $\$ 240,000$ & \\
$25 \%$ & $\$ 180,000$ & $\$ 0$ \\
$20 \%$ & $\$ 120,000$ & $\$ 60,000$ \\
$15 \%$ & $\$ 60,000$ & $\$ 120,000$ \\
$0 \%$ & $\$ 0$ & $\$ 180,000$ \\
\hline
\end{tabular}

Task 2

\begin{tabular}{lrr}
\hline Issue & Total value to Bainbridge & Total value to Alki \\
\hline $\begin{array}{l}\text { Building inspector } \\
\text { Conibear }\end{array}$ & $\$ 80,000$ & \\
Hawes & $\$ 60,000$ & $\$ 80,000$ \\
Gillespie & $\$ 40,000$ & $\$ 60,000$ \\
DeWitt & $\$ 20,000$ & $\$ 40,000$ \\
Wottle & $\$ 0$ & $\$ 20,000$ \\
Height of building & & $\$ 0$ \\
2 stories & $\$ 120,000$ & \\
3 stories & $\$ 90,000$ & $\$ 0$ \\
4 stories & $\$ 60,000$ & $\$ 90,000$ \\
5 stories & $\$ 30,000$ & $\$ 180,000$ \\
6 stories & $\$ 0$ & $\$ 270,000$ \\
& & $\$ 360,000$ \\
\hline
\end{tabular}

\section{References}

Argyris, C. 1993. On Organizational Learning. Blackwell, Cambridge, MA.

Balke, W. M., K. R. Hammond, G. D. Meyer. 1973. An alternate approach to labor-management relations. Admin. Sci. Quart. 18(3) 311-327.

Ball, S. B., M. H. Bazerman, J. S. Carroll. 1991. An evaluation of learning in the bilateral winner's curse. Organ. Behavior Human Decision Processes 48 1-22.

Balzer, W., M. Doherty, R. O'Connor. 1989. Effects of cognitive feedback on performance. Psych. Bull. 106 110-433.

Bandura, A. 1973. Aggression: A Social Learning Analysis. PrenticeHall, Englewood Cliffs, NJ.

Bazerman, M. H., J. J. Gillespie. 1999. Betting on the future: The virtues of contingent contracts. Harvard Bus. Rev. 77(4) 155-160.

— M. A. Neale. 1982. Improving negotiation effectiveness under final offer arbitration: The role of selection and training. J. Appl. Psych. 67(5) 543-548.

Bryan, J. H., M. A. Test. 1967. Models and helping: Naturalistic studies in aiding behavior. J. Personality Soc. Psych. 6 400-407.

Cook, T. D., D. T. Campbell. 1979. Quasi-Experimentation: Design and Analysis Issues for Field Settings. Rand McNally, Chicago, IL.

Couzijn, M. 1999. Learning to write by observation of writing and dreading processes: Effects on learning and transfer. Learn. Instruction 9 109-142.

Decety, J., J. Grezes, N. Costes, D. Perani, M. Jeannerod, E. Procyk, R. Grassi, F. Fazio. 1997. Brain activity during observation of actions: Influence of action content and subject's strategy. Brain 120(10) 1763-1777.

Donnelly, C. M., M. A. McDaniel. 1993. Use of analogy in learning scientific concepts. J. Experiment. Psych: Learn. Memory, Cognition 19 975-987.

Einhorn, H., R. Hogarth. 1978. Confidence in judgment: Persistence of the illusion of validity. Psych. Rev. 85(5) 395-416.

Gentner, D. 1983. Structure-mapping: A theoretical framework for comparison. Cognitive Sci. 7 155-170. 
1989. The mechanisms of analogical learning. S. Vosniadou, A. Ortony, eds. Similarity and Analogical Reasoning. Cambridge University Press, New York, 199-241.

_ , M. J. Rattermann, K. D. Forbus. 1993. The roles of similarity in transfer: Separating retrievability from inferential soundness. Cognitive Psych. 25(4) 524-575.

Gick, M. L., K. J. Holyoak. 1983. Schema induction and analogical transfer. Cognitive Psych. 15 1-38.

Hammond, K. R., G. H. McClelland, J. Mumpower. 1980. Human Judgment and Decision Making. Praeger, New York.

Hogarth, R. M. 1981. Beyond discrete biases: Functional and dysfunctional aspects of judgmental heuristics. Psych. Bull. 90(2) 197-217.

Holyoak, K. J. 1985. The pragmatics of analogical transfer. G. H. Bower, ed. The Psychology of Learning and Motivation, Vol. 19. Academic Press, New York, 59-87.

— P. R. Thagard. 1989. Analogical mapping by constraint satisfaction. Cognitive Sci. 13 295-355.

— - 1995. Mental Leaps: Analogy in Creative Thought. MIT Press, Cambridge, MA.

- -1997 . The analogical mind. Amer. Psych. 52 35-44.

Loewenstein, J., L. Thompson, D. Gentner. 1999. Analogical encoding facilitates transfer in negotiation. Psychonomic Bull. Rev. 6(4) 586-597.

Neale, M. A., G. B. Northcraft. 1990. Behavioral negotiation theory: A framework for conceptualizing dyadic negotiation. L. L. Cummings, B. Staw, eds. Research in Organizational Behavior. JAI Press, Greenwich, CT.

Novick, L. R. 1988. Analogical transfer, problem similarity, and expertise J. Experiment. Psych: Learn. Memory, Cognition. 14(3) 510-520.

Pirolli, P. L., J. B. Anderson. 1985. The role of learning from exam- ples in the acquisition of recursive programming skills. Canadian J. Psych. 39 240-272.

Platt, J. R. 1964. Strong inference. Science 146 347-353.

Polanyi, M. 1958. Personal Knowledge: Towards a Post-Critical Philosophy. Routledge and Kegan Paul, London, U.K.

Polya, G. 1957. How to Solve it, 2nd ed. Doubleday, New York.

Ross, B. H. 1987. This is like that: The use of earlier problems and the separation of similarity effects. J. Experiment. Psych.: Learn. Memory, Instruction 13 629-639.

_ M. C. Kilbane. 1997. Effects of principle explanation and superficial similarity on analogical mapping in problem solving. J. Experiment. Psych.: Learn, Memory, Cognition 23(2) 427-440.

Shebilske, W. L., J. A. Jordon, B. P. Goettle, L. E. Paulus. 1998. Observation versus hands-on practice of complex skills in dyadic, triadic, and tetradic training-teams. Human Factors 40(4) 525-540.

Spector, B. I. 1997. Analytical support to negotiations: An empirical assessment. Group Decision Negotiation 6(5) 421-436.

Thompson, L. 1990a. The influence of experience on negotiation performance. J. Experiment. Soc. Psych. 26(6) 528-544.

. 1990b. An examination of naive and experienced negotiators. J. Personality Soc. Psych. 59(1) 82-90.

_, T. DeHarpport. 1994. Social judgment, information feedback, and interpersonal learning in negotiation. Organ. Behavior Human Decision Processes 58(3) 327-345.

_, R. Hastie. 1990. Social perception in negotiation. Organ. Behavior Human Decision Processes 47(1) 98-123.

_, J. Loewenstein, D. Gentner. 2000. Avoiding missed opportunities in managerial life: Analogical training more powerful than case-based training. Organ. Behavior Human Decision Processes 82(1) $60-75$.

Accepted by Linda Argote, William McEvily, and Ray Reagans; received February 28, 2001. This paper was with the authors 6 months for 2 revisions. 